\title{
Study on Characteristics of Stress Relaxation for Typical Metallic Sealing Materials
}

\author{
Jian-Chun DING ${ }^{1, a}$, Chuan-Jun LIAO ${ }^{2, b,}$, Ting ZHANG ${ }^{2}$, Kai WANG ${ }^{2}$ \\ ${ }^{1}$ School of Aeronautics, Northwestern Polytechnical University, Xian 710072, China \\ ${ }^{2}$ Beijing Institute of Astronautical Systems Engineering, Beijing 100076, China \\ a13331001339@163.com, ${ }^{b}$ liaochuanjunl@163.com \\ * Corresponding author
}

Keywords: Metallic seal, Aluminum, Silver, Stress Relaxation, Dynamic equation.

\begin{abstract}
The stress relaxation of the metallic seal is a general problem during its long-term service, which is harmful for the sealing performance. For the metallic seal, the sealing mechanism and the problem of the stress relaxation are analyzed, and the research methods applied to study the problem of stress relaxation of the metallic sealing material are proposed in this paper. Further, the dynamic equation of the metallic seal is developed, and the data processing method and the solving method of the dynamic equation are also put forward, which are based on the stress relaxation experiment. Based on the proposed mathematics methods and the experimental data of the stress relaxation, the analysis about the characteristics of stress relaxation of the two metallic sealing materials are carried out, which include of the aluminum and the silver that are common materials used by the metallic seal. To the specific test conditions, the dynamic equations of stress relaxation are respectively presented, which lay the foundation for judging the sealing performance of the metallic seal during its long-time service.
\end{abstract}

\section{Introduction}

More and more equipments require its metallic seals to service for several decades, which can help to improve its security, reliability and service efficiency. However, it is true that the metallic seal will generate stress relaxation during its long-time service, which is unfavorable for its sealing performance. So, it is required to study the characteristics of stress relaxation of metallic seals that are favorable to complete the design, improve the anti-stress relaxation ability and predict the operating time of the metallic seal, and the studies about the mechanisms, characteristics and influencing factors should be carried out [1].

Based on the studies about the sealing mechanism and the problem of the stress relaxation of the metallic seal, a mathematical method used to study the stress relaxation of metallic seal is proposed in this paper, and the characteristics of stress relaxation for some typical metallic sealing materials are presented.

\section{Sealing Mechanism and Stress Relaxation of Metallic Seal}

\subsection{Sealing Mechanism of Metallic Seal}

By the pretightening load, the metallic seal is compressed, and the resilience force generated by the compression can make the surface material of the metallic seal yield. Then, the plastic flow of the surface material fills the microcosmic leakage paths on the sealing surface that can be considered to be closed when the yield rate is sufficient. Therefore, the sealing requirement of the medium can be satisfied. The sealing mechanism of the metallic seal is as shown in Fig. 1. When the pretightening load doesn't apply to the sealing surface, there are only some contacts of roughness peaks, and a large number of leakage paths exist on the sealing surface, as shown in Fig. 1(a). As the load increases, both the contact number and the deformations of the roughness peaks increase, and the surface material of the metallic seal begins to produce plastic deformation. When the load increases further, the surface material yield obviously, and the plastic flow is sufficient to fill in the gaps on 
the sealing surface, so the leakage of the medium can be prevented, as shown in Fig.1 (b) [2].

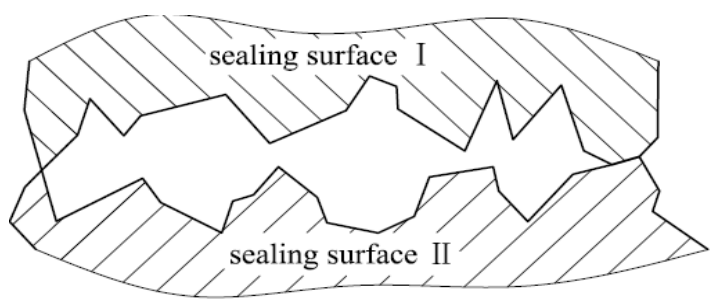

(a) initial concact state before loading

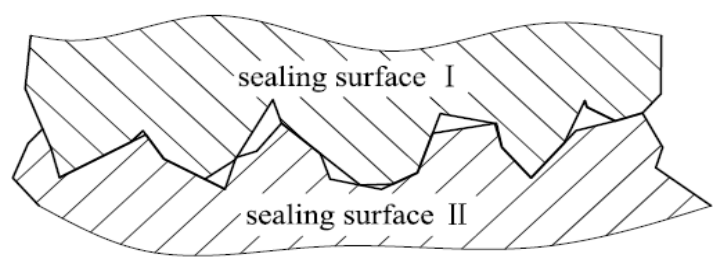

(b) concact state after loading

Fig. 1 Diagrammatic sketch of the sealing mechanism of metallic seals

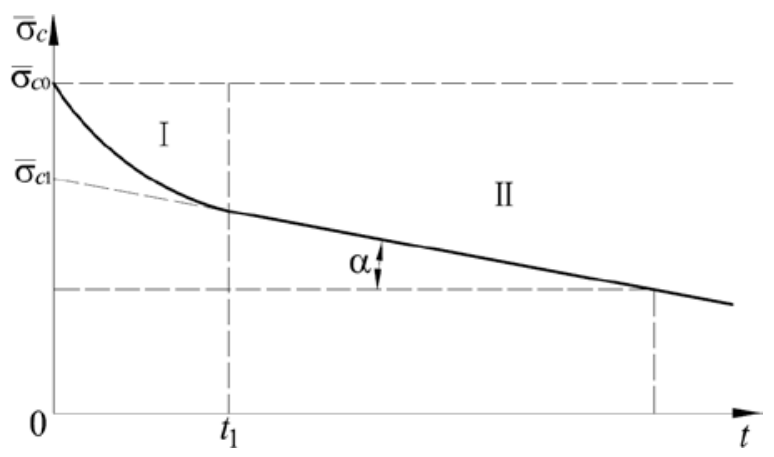

Fig. 2 A typical stress relaxation curve of the metallic seal material

\subsection{Stress Relaxation of Metallic Seal}

For the metallic seal of long-time use, the stress relaxation greatly affects the sealing performance. The stress relaxation of the metallic seal is mainly caused by the following factors. One is related with the metallic seal itself. As the service time increases, both the structural stress and the contact stress of the metallic seal decreases, and the leakage rate might be above the allowable value. The other is related with the joint structure and the fastening pieces that there exit the problem of stress relaxation similarly, which can make the sealing load decrease and lead the leakage rate to exceed the allowable value. Therefore, it is very important to systematically study the characteristics of stress relaxation of the metallic seal, and the effects of the stress relaxation on the sealing performance should be minimized [2].

\section{Research Technique of Stress Relaxation of Metallic Seal}

\subsection{Definition of Stress Relaxation}

When the strain is invariant under both the stationary temperature and the stationary initial load, the stress relaxation is defined as the phenomenon that the stress of the metal material or its hardware decreases with the time expands. The stress relaxation is a general phenomenon, and it is one of the key factors that lead the metallic seal of long-time service to fail. So, the studies about the stress relaxation are very important, including of its regularity, character and influencing factors etc. These are favorable for selecting the material, improving the anti-stress relaxation performance, forecasting the operation life of the metallic seal [3].

\subsection{Curve and Index of Stress Relaxation}

Under the isothermal condition, a typical stress relaxation curve of the metallic seal material is shown in Fig. 2, and $\bar{\sigma}_{c}$ is defined as the equivalent stress. According to the variation trend, the curve can be divided into two stages. For the stage I, the time of duration is quite short and the equivalent stress rapidly decreases with time. The initial stress $\bar{\sigma}_{c 0}$ takes significant effect on the descending speed the stress. Practically, it is impossible to investigate the stage I by experimental methods. For the stage II, the endurance period is very long, and the stress gradually reduces with time and approaches to one constant value. The stress relaxation characteristics of this stage lay foundation for the analysis of the stress relaxation of the metallic seal [4].

For the stage II, the relation of the equivalent stress with the time can be thought to be linear, and 
the stress relaxation rate with small $\alpha$ shows good stability for the metallic seal. $\bar{\sigma}_{c 1}$ is defined as the initial stress of the stage II, both $\bar{\sigma}_{c 1}$ and $\alpha$ can be obtained by the stress relaxation experiment of the metallic seal material.

\subsection{Influencing Factors of Stress Relaxation}

It is very complicated for the influencing factors of the stress relaxation of metal materials, which are divided into the intrinsic factors and the extrinsic factors. The extrinsic factors include of the load, the time and the environmental temperature, and the intrinsic factors include of the chemical constitution, the plastic deformation, the metallurgical structure and the mechanical performance etc. It is well known that there are many crystal defects in the interior of the metal material, and the inner energy is higher. These cause its metallurgical structure and dislocation substructure are instability. Under some definite conditions including of the load, temperature and actuation duration, the above status will transform to the status of low free energy, and some elastic deformations change to the plastic deformation and some storage energies are delivered. The mentioned above phenomenon can be regarded to be the stress relaxation course of the metal material or its product.

Generally, the extrinsic influencing factors of stress relaxation for metallic seal materials are divided into three classes that are individually the time relaxation, the load relaxation and the temperature relaxation [5].

The stress relaxation rate $v_{s}$ is related with the time, and the relationship can be expressed by

$$
v_{s}=a_{1}+b_{1} \ln t
$$

Where $a_{1}$ and $b_{1}$ are the constants that are decided by the species and the test conditions of the metallic seal material.

The stress relaxation rate is related with the stress level, and the expression is

$$
v_{s}=a_{2} e^{b_{2} \tau}-c_{1}
$$

Where $\tau$ is the index of the stress level, and $a_{2}, b_{2}$ and $c_{1}$ denote the constants related with the material attribute and the experimental conditions.

The stress relaxation rate is related with the time, which can be expressed by

$$
v_{s}=a_{3} e^{-Q / k T}
$$

Where $Q$ expresses the activation energy in the stress relaxation course of the metallic sealing material. Besides $a_{3}$ is a constant and $k$ is the Boltzmann's constant.

For the temperature relaxation, the stress relaxation rate of the metallic sealing material increases with the temperature increment and decreases with the increment of the activation energy. Some studies show that some alloy agents are favorable for the improvement of the activation energy, such as $\mathrm{Cr}, \mathrm{Si}, \mathrm{Ni}, \mathrm{W}, \mathrm{Nb}$ and $\mathrm{V}$ etc.

\subsection{Dynamic Equation of Stress Relaxation}

The basic expression of the dynamic equation of stress relaxation for the metal material is [6]

$$
\sigma_{\mathrm{sh}}=\sigma_{0}-v_{\mathrm{s}} \ln t
$$

Where $\sigma_{\text {sh }}$ and $\sigma_{0}$ respectively show the residual stress and the initial stress.

The upper expression can be expressed by the loss factor of the load, and its expression is

$$
\frac{\Delta P}{P_{0}} \times 100 \%=a+b \ln t
$$

Where $P_{0}$ and $\Delta P$ respectively denote the initial load and the loss load. Besides, both $a$ and $b$ are the constants that are decided by the metal material and the experimental condition. 


\section{Stress Relaxation Characteristic of Typical Metallic Sealing Materials}

\subsection{Mathematical Methods}

According to Esq. (4), the dynamics equation of stress relaxation for metallic sealing material is defined as

$$
Y_{2 \mathrm{R}}(T, t)=K(T)-C(T)\left(\log _{10}{ }^{(t)}\right)
$$

Where $Y_{2 \mathrm{R}}(T, t)$ represents the residual stress. $K(T)$ and $C(T)$ are the constants related with the metal material and the testing conditions, which can be obtained by the statistical analysis of the test data. So the dynamic equation can be determined. Using this equation, the residual stress of the metallic seal at any time can be forecasted, which can provide instructions for the judgment of the sealing performances.

By the experiment of stress relaxation of the metallic sealing material, the measured data $\left(\log _{10}{ }^{\left(t_{i}\right)}\right.$, $Y_{2 R_{1}}$ ) has been obtained. It is hypothesized that the error of the independent variable can be ignored, so the following expressions can be defined.

$$
X_{i}=\log _{10}{ }^{\left(t_{i}\right)} D=\sum_{i=1}^{n}\left[Y_{2 R_{i}}-K-C X_{i}\right]^{2}
$$

The first-order partial derivative of $D$ to $K$ and $C$ respectively are as follows.

$$
\frac{\partial D}{\partial K}=-2\left[\sum_{i=1}^{n} Y_{2 R_{i}}-n K-C \sum_{i=1}^{n} X_{i}\right] \frac{\partial D}{\partial C}=-2\left[\sum_{i=1}^{n} Y_{2 R_{i}} X_{i}-K \sum_{i=1}^{n} X_{i}-C \sum_{i=1}^{n}\left(X_{i}\right)^{2}\right]
$$

Further, the second-order partial derivatives are respectively obtained by

$$
\frac{\partial^{2} D}{\partial K^{2}}=2 n \geq 0 \frac{\partial^{2} D}{\partial C^{2}}=2 \sum_{i=1}^{n}\left(X_{i}\right)^{2} \geq 0
$$

It can be concluded that the condition of the minimal value can be satisfied. If the first-order partial derivative equal to zero, the following expressions can be obtained.

$$
\sum_{i=1}^{n} Y_{2 R_{i}}-n K-C \sum_{i=1}^{n} X_{i}=0 \sum_{i=1}^{n} Y_{2 R_{i}} X_{i}-K \sum_{i=1}^{n} X_{i}-C \sum_{i=1}^{n}\left(X_{i}\right)^{2}=0
$$

The calculations of the average values are introduced into the analysis, and the following average values are defined.

$$
\begin{aligned}
& \bar{X}=\frac{1}{n} \sum_{i=1}^{n} X_{i} \quad \bar{Y}_{2 R}=\frac{1}{n} \sum_{i=1}^{n} Y_{2 R_{i}} \\
& \overline{X^{2}}=\frac{1}{n} \sum_{i=1}^{n} X_{i}^{2} \quad \overline{X Y_{2 R}}=\frac{1}{n} \sum_{i=1}^{n} X_{i} Y_{2 R_{i}}
\end{aligned}
$$

Further

$$
\bar{Y}_{2 R}-K-C \bar{X}=0 \quad \overline{X Y_{2 R}}-K \bar{X}-C \overline{X^{2}}=0
$$

So, the constants in the dynamic equation can be solved, and are expressed by

$$
K=\bar{Y}_{2 R}-C \bar{X} \quad C=\frac{\overline{X Y_{2 R}}-\bar{X} \bar{Y}_{2 R}}{\overline{X^{2}}-(\bar{X})^{2}}
$$

When the values of $K$ and $C$ are substituted into the dynamic equation, the liner regression equation can be obtained.

The standard deviations of $Y_{2 \mathrm{R}}, K$ and $C$ are respectively calculated by 


$$
\begin{gathered}
\sigma_{Y_{2 R}}=\sqrt{\frac{\sum_{i=1}^{n}\left[Y_{2 R_{i}}-K-C X_{i}\right]^{2}}{n-2}} \\
\sigma_{K}=\sqrt{\frac{\overline{X^{2}}}{n\left(\overline{X^{2}}-(\bar{X})^{2}\right)} \sigma_{Y_{2 R}} \quad \sigma_{C}=\sqrt{\frac{1}{n\left(\overline{X^{2}}-(\bar{X})^{2}\right)}} \sigma_{Y_{2 R}}}
\end{gathered}
$$

So, the correlation coefficient can be calculated by

$$
C_{1}=\frac{\overline{X Y_{2 R}}-\bar{X} \bar{Y}_{2 R}}{\sqrt{\left(\overline{X^{2}}-(\bar{X})^{2}\right)\left(\overline{Y_{2 R}^{2}}-\left(\overline{Y_{2 R}}\right)^{2}\right)}}
$$

Based on the liner regression equation, the uncertainty of the estimated value $Y_{2 \mathrm{R}}$ can be calculated, and the expression is as follows.

$$
u_{c}\left(Y_{2 R}\right)=\sigma_{Y_{2 R}} \cdot \sqrt{\frac{1}{m}+\frac{1}{n}+\frac{\left(\log _{10}{ }^{(t)}-\bar{X}\right)^{2}}{\sum_{i=1}^{n}\left(\log _{10}{ }^{\left(t_{i}\right)}-\bar{X}\right)^{2}}}
$$

Where $m$ represents the measurement number of the experimental value.

It is defined that $\alpha$ is the confidence interval and $k$ is the inclusion factor, and the extended uncertainty can be further calculated by

$$
U_{c}\left(Y_{2 R}\right)=k u_{c}\left(Y_{2 R}\right)
$$

So, $Y_{2 \mathrm{R}}$ can be calculated by the following equation when the uncertainty is

$$
Y_{2 R}(t)=K-C \log _{10}{ }^{(t)}-U_{c}\left(Y_{2 R}\right)
$$

\subsection{Characteristic Analysis of Stress Relaxation}

The aluminum is one of the major materials used by metallic seals, and the main geometric and operating parameters of the studied aluminum specimen is shown in table $1[7,8]$.

Table 1 Main geometric and operating parameters

\begin{tabular}{|l|l|l|l|}
\hline Symbol & Notation & Value & Unit \\
\hline$h$ & Thickness & 0.4 & $\mathrm{~mm}$ \\
\hline$r$ & Inside radius & 4.1 & $\mathrm{~mm}$ \\
\hline$R$ & Outer radius & 6.1 & $\mathrm{~mm}$ \\
\hline$T$ & Temperature & 200 & ${ }^{\circ} \mathrm{C}$ \\
\hline
\end{tabular}

Table 2 Main geometric and operating parameters

\begin{tabular}{|l|l|l|l|}
\hline Symbol & Notation & Value & Unit \\
\hline$h$ & Thickness & 0.3 & $\mathrm{~mm}$ \\
\hline$r$ & Inside radius & 4.2 & $\mathrm{~mm}$ \\
\hline$R$ & Outer radius & 6.2 & $\mathrm{~mm}$ \\
\hline$T$ & Temperature & 100 & ${ }^{\circ} \mathrm{C}$ \\
\hline
\end{tabular}




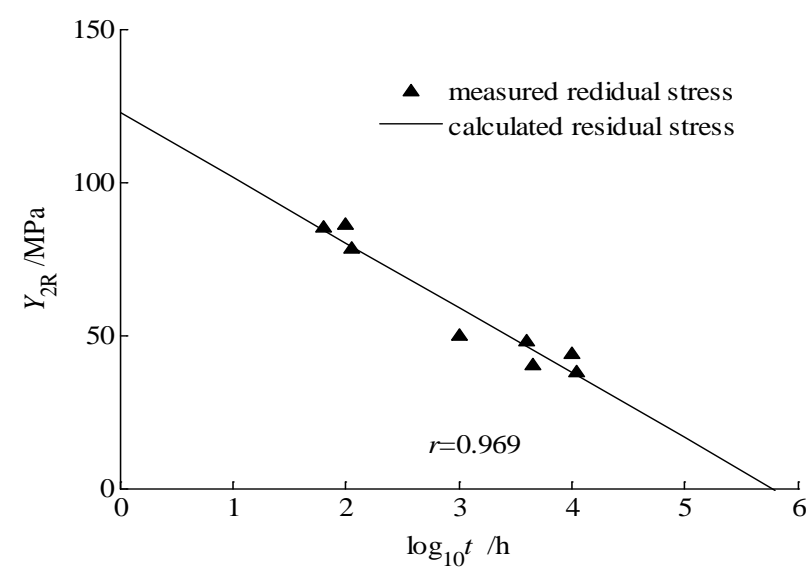

Fig. 3 Experimental data and its fitting curve of the aluminum specimen

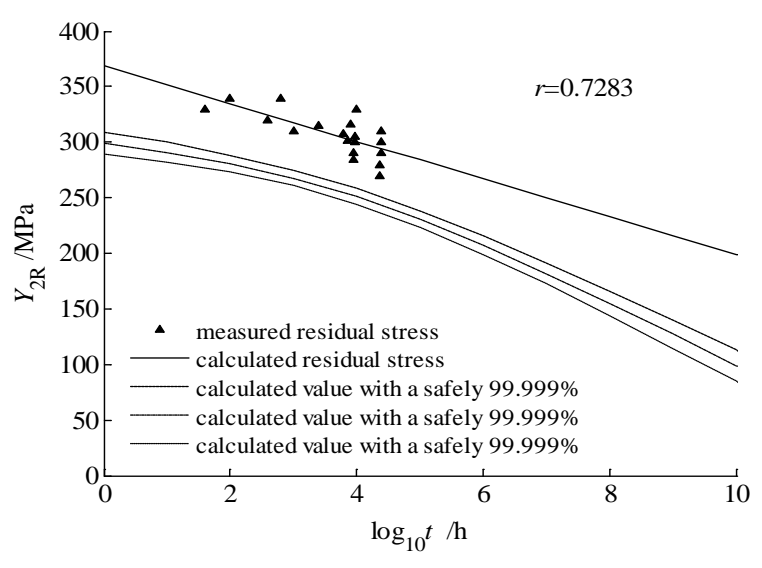

Fig. 4 Experimental data and its fitting curve of the silver specimen

The experimental data and its fitting curve of the studied specimen are shown in Fig. 3 [7]. Therefore, the dynamic equation is obtained and is expressed by

$$
Y_{2 R}=122.7342-21.237\left(\log _{10}{ }^{(t)}\right)
$$

The silver is also one of the major materials used by metallic seals, and the main geometric and operating parameters of the studied silver specimen is shown in table 2 [7]. The experimental data and its fitting curve of the studied specimen are shown in Fig. 4 [7]. So, the dynamic equation is obtained and is expressed by

$$
Y_{2 R}=369.027-17.0355\left(\log _{10}{ }^{(t)}\right)
$$

\section{Conclusions}

By the studies of the sealing mechanism, the stress relaxation and the relations between the above two contents, the research methods applied to study the problem of the metallic sealing material are proposed, which help to disclosure the inner characteristic and main influencing factors of the stress relaxation of the metallic seal. The dynamic equation of stress relaxation for the metallic seal is developed, and its mathematical processing methods are presented at the same time. So, the characteristics of stress relaxation of the studied aluminum, silver materials are presented, and the dynamic equations are respectively obtained. These can provide the instructions for the judgments of the sealing performances of the metallic seals during the long-time service

\section{Acknowledgement}

The authors acknowledge the financial support by the Project study on behavior of stress relaxation and leakage model of metal seal applied to corrosive medium supported by National Natural Science Foundation of China (No. 51305040).

\section{References}

[1] B.CH. Wen, Handbook of mechanical design, Mechanical Industry Press, Beijing, 1994.

[2] CH.J Liao, D.L. Wang, H.R. Wang, et al, Features and applications of typical non-conventional type metal seals, Cryogenics. 20(2014)55-60.

[3] D.D. Su, Stress relaxation and stabilizing treatment of steel wire products, Steel Wire Products. 26(2000)1-6.

[4] D.D. Su, ZH.SH. Zhu, X.H. Wang. Research on anti-stress relaxational ability of helical compression springs made of austenitic stainless steel wires, Steel Wire Products. 23(1997)12-18. 
[5] D.D. Su. Spring (material) stress relaxation and its prevention, Tianjing University Press, Tianjing, 2000.

[6] D.D. Su, F. Wu, Y.L. Li, et al, Stress relaxation treatment of the leaf spring made of maraging stainless steel and prediction of its relaxational failure lifetime, Journal of Tianjing University. 31(1998)234-240.

[7] H. Sassoulas, L. Morice, P. Caplain, et al, Ageing of metallic gaskets for spent fuel casks: Century-long life forecast from 25,000-h-long experiments, Nuclear Engineering and Design. 236(2006)2411-2417.

[8] Q. Chen, L.CH. You, L.X. Shi, et al, Characteristic study of stress relaxation on V-type metallic spiral wound gasket, Lubrication and Sealing. 23(2003)54-55. 